\title{
Secondary reinforcing properties of informative and non-informative stimuli'
}

BENJAMIN MCKEEVER, UNIVERSITY OF WASHINGTON

BERT FORRIN, SCARBOROUGH COLLEGE, UNIVERSITY OF TORONTO

This study compared the secondary reinforcing properties of two cues distinguished on the basis of their prior significance in a brightness discrimination problem: a cue discriminative for reinforcement but not present in the goal region (a non-redundant predictor of reward) and a cue present in the goal region but irrelevant to the acquisition of the required discrimination (a redundant predictor or reward). The findings suggest that both cues were equally effective in reinforcing a simple position habit.

A revision of the traditional Hullian conception of secondary reinforcement (Hull, 1943) has recently been proposed by Egger \& Miller $(1962,1963)$. They assert that the potency of a conditioned reinforcer is influenced not solely by its proximity to primary reward but by its informative properties. Given a sequence of stimuli which invariably precede a reinforcing eventby a brief (but unspecified) span of time, only the first stimulus is a non-redundant predictor of reward-to-come. Later cues in the sequence add no new information relative to the occurrence of reinforcement. Egger and Miller contend that, by virtue of its information value, the first stimulus, despite the greater delay of primary reinforcement, is most likely to acquire secondary reward value. Their experimental findings were consistent with this position. The present investigation serves to provide a further test of the Egger-Miller information hypothesis.

Method

The Ss were 32 experimentally naive, male albino rats of the Sprague-Dawley strain, approximately 110 days old. Ss were run in two squads (replications) of 16 animals each. A two-stage procedure was employed.

1. Brightness discrimination training.-All $\mathrm{Ss}, 23 \mathrm{hr}$. hungry, received 10 trials per day in the modified Grice maze shown in Fig. 1. Half were trained on the greyblack (black positive) problem illustrated; the remainder, on a grey-white (white positive) problem. In the former case, the antechamber leading to the positive goal box and its entrance and exit doors were flat black; the goal chamber was flat white. In the latter, the positive discriminative cue was white and the positive goal box, black. The remainder of the maze, except for the clear Plexiglas start-box door, was painted flat mid-grey. All doors were of the swinging type with stops to prevent retracing. A correct choice was reinforced by a pellet of Gaines' Dog Meal. Criterion performance was defined as at least nine correct responses on each of two successive days. Each $\mathrm{S}$ re-
A. PLAN FOR TRAINING MAZE

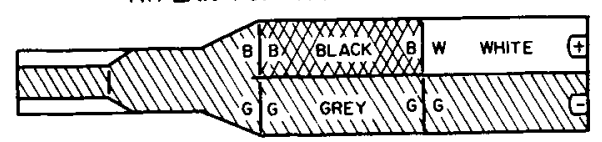

B. PLAN FOR TEST MAZES

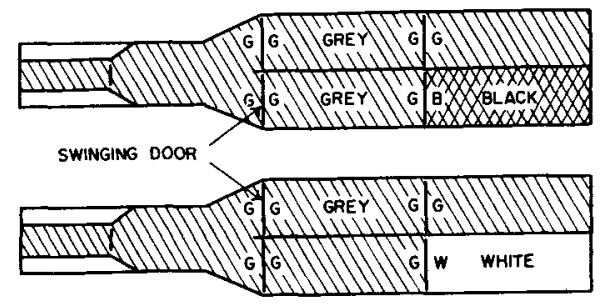

Fig. 1. Sample floor plans for the brightness discrimination training and the position habit test mazes. $(.1$ in. $=1$ in. $)$. The brightness for each side of a swinging door is given by the letters B (black), G (grey), and $W$ (white). For the training maze indicated, the critical alley in the upper test maze terminates in the prior discriminative cue; the critical alley in the lower test maze terminates in the prior goal-box cue.

ceived two additional post-criterion days of training before being shifted to the position problem.

2. Position habit training.-Training was conducted in a slightly altered version of the discrimination apparatus. The form of the maze was unchanged but, with the exception of one goal box, the entire maze was painted mid-grey. For one-half of the Ss, the brightness of one goal box (designated the critical cue) was that of the previous discriminative cue; for the remainder, the brightness was that of the goal chamber during discrimination training. Since the effect of the critical cue upon position preference was at issue, its location was fixed for each $\mathrm{S}$ throughout training. The brightness of the critical cue (black or white) and its location (left or right) were completely counterbalanced over Ss.

All Ss were $23 \mathrm{hr}$. hungry when trained. A noncorrection procedure was employed. No food reinforcement was offered but Ss were permitted to remain in the chosen goal box for 15 sec. Training was conducted on two successive days. On the first, Ss were initially given one free trial followed by one forced trial to the side not chosen. All subsequent trials were free. Ss received eight additional trials on the first day and 10 on the second.

\section{Results}

Mean per cent choice of the critical alley is plotted as a function of successive blocks of two trials in Fig. 2. With Trials 1 and 2 omitted, the data were analyzed in terms of a Conditions by Replications by Days design. 


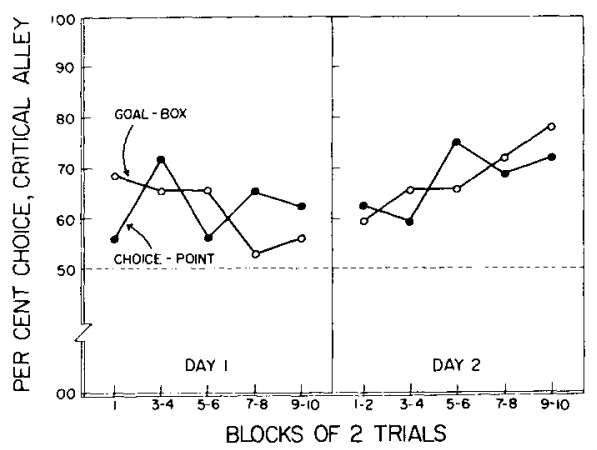

Fig. 2. Per cent choice of the critical alley as a function of blocks of two trials for the two experimental groups. Data for the second trial on Day 1 (forced) are omitted.

No main or interaction effects proved statistically significant. Preference for the alley terminating in the prior choice-point cue did not differ significantly from preference for the alley terminating in the prior goalbox cue $-F<1, d f=1 / 28$. Nor was there a significant change in preference over conditions from the first to the second day of training $-F=1.141, \mathrm{df}=1 / 28$. A more fine grain analysis in terms of Conditions by Replications by Blocks within Days yielded only one statistically significant effect: preference for the critical side increased linearly over blocks on Day $2-F=7.480$, $\mathrm{df}=4 / 112, \mathbf{p}<.01$. Though, as noted, variation in the prior role of the critical cue had no significant effect upon performance, the mean per cent runs to the critical side $(65.97 \%$ for the choice-point group, $64.58 \%$ for the goal-box group) significantly exceeded the a priori chance base $(50 \%)$ in each condition $-t=2.733, \mathrm{df}=15$, $p<.02$ and $t=3.121, d f=15, p<.01$, respectively.

\section{Discussion}

The test for secondary reinforcement was a stringent one involving the capacity of the critical cues to facilitate the learning of a new position habit in the absence of primary reinforcement. In addition, insofar as the critical cues were not present at the choicepoint in the test maze, their effect is to be attributed not to their response-eliciting properties but to their reinforcing properties. The choice of critical alley with frequency greater than that expected by chance would, therefore, seem to establish the acquired reward value of both the prior choice-point cue and the prior goalbox cue.

There are several grounds for caution in the unqualified acceptance of the latter conclusion. It is possible that position preference was influenced by aversive properties conditioned to the grey (formerly negative) goal chamber rather than by the acquired reward value of the critical cues. Or, the effective determinant of choice behavior may have been the change in brightness associated with the critical alley. Further, the evidence for the anticipated increase in preference for the critical side over training is ambiguous.

These limitations aside, if the occurrence of secondary reinforcement may be granted, it is apparent that the magnitude of the reinforcing effect did not differ between choice-point and goal-boxcues. Consistent with the views of Egger and Miller, no gradient effect was noted. Though in greater temporal proximity to food reinforcement during brightness discrimination training, the goal-box cue did not acquire a greater reinforcing capacity. But, its considerable conceptual appeal notwithstanding, the Egger-Miller (1962) hypothesis that "a redundant predictor of primary reinforcement should not acquire secondary reinforcement strength" "was not substantiated by the present data. Both critical cues were equally reliable predictors of primary reward but the goal-box cue was completely redundant. Ss could and did predict the location of primary reinforcement from the choice-point cue alone. Yet this difference in information value did not conduce to a difference in acquired reward value.

\section{References}

Egger, M. D., \& Miller, N. E. Secondary reinforcement in rats as a function of information value and reliability of the stimulus. $J$. exp. Psychol., 1962, 64, 97-104.

Egger, M. D., \& Miller, N. E. When is a reward reinforcing?: an experimental study of the information hypothesis. $J$. comp. physiol. Psychol., 1963, 56, 132-137.

Hull, C. L. Principles of behavior. New York: Appleton-Century, 1943

Note

1. Supported in part by a grant from the Graduate School Research Fund of the University of Washington. The authors are indebted to Sharon Waite and Kelly Shaver for their assistance in the execution of this study. 